\title{
MOISTURE TRANSPORT IN POLYAMIDE (PA6) AND ITS EFFECT ON THE MECHANICAL BEHAVIOUR
}

\author{
PRATEEK SHARMA ${ }^{1}$, STEFAN DIEBELS ${ }^{1}$ \\ ${ }^{1}$ Chair of Applied Mechanics \\ Campus A4 2, 1. OG \\ 66123 Saarbrücken \\ prateek.sharma@uni-saarland.de \\ https://www.uni-saarland.de/lehrstuhl/diebels.html
}

Key words: Coupled Model, Non-Linear Diffusion, Viscoelastic, Multiphysics

\begin{abstract}
Polyamide exhibits hygroscopic nature and can absorb up to $10 \%$ of moisture relative to its weight. The absorbed moisture increases the mobility of the molecular chains and causes a reduction in the glass transition temperature. Thus, depending on the moisture distribution, a polyamide component can show different stiffness and relaxation times.

The moisture distribution also depends on the mechanical loading of the material. However, it was noticed that the diffusion process remains unaffected when the process is compared for a loaded and an unloaded material. It is postulated that the moisture redistribution is due to an external force that takes place as a result of the pressure acting on the moisture. The diffusion process is unaffected by preloading of the dry material as the pressure is applied purely on the material and not on the absorbed moisture. However, when a saturated specimen is loaded, the pressure is exerted on the moisture too which causes its redistribution.

In this work, the distribution of the absorbed moisture is simulated by a non-linear diffusion model. It is coupled with the viscoelastic behaviour of PA6. The stiffness of the viscoelastic model changes and the relaxation time reduces with increasing moisture concentration. The coupling of diffusion to mechanical loading is achieved with the recalculation of the moisture concentration caused due to the redistribution of volume. It is assumed that there is no transport of moisture, but the transport of volume and the change in volume creates a change in concentration in the specimen. This strongly coupled model has been implemented using the finite element method. The model results are compared to experiments for validation. A strongly coupled model was thus created which could reproduce the experimental results with reasonable accuracy.
\end{abstract}

\section{INTRODUCTION}

The use of polymer has increased continuously in the last decades. In the last 50 years, the consumption of polymer has increased from 5 million ton to 100 million ton worldwide [22]. Technical polymers such as polyamide (PA6) form a major portion of the polymer applications. Lightweight, low cost and manufacturability are some of the reasons why PA6 is used more often. Apart from that, its chemical resistance is useful in applications such as in the automobile industry where they are used in fuel supply components, air intake manifolds and engine covers [5, 23-25]. However, PA6 is used under various 
environmental conditions, where the temperature and humidity can vary with time. As it is hygroscopic in nature, it can absorb from $9.5 \%$ to $10 \%$ of moisture by weight $[1,2,9]$. The diffusion of water in the polyamide depends on various parameters such as temperature, loading conditions and time. When the surrounding humidity varies polyamide can absorb water at high relative humidities but due to plastification, it can again give up some moisture causing an in-stationary and inhomogeneous distribution of moisture in the material $[4,8,10,16]$. In the industry however, the local distribution of moisture is not taken into account and rather a saturated and a dry material is studied. The presence of moisture can increase the PA6 chain mobility altering its mechanical behaviour $[6,7,17]$. Further, the moisture uptake behaviour is also effected by the loading as the free volume available for moisture absorption changes. In order to model the behaviour of PA6 under the presence of moisture, it is important to understand the effect of water on the mechanical properties as well as the effect of mechanical loading on the diffusion process. The water molecules can form hydrogen bonds with the polymer chains [19], which causes the gliding of the chains against each other. This leads to a transformation from a glassy state to a rubbery state, thus lowering the glass transition temperature [13]. One of the effects of the phase transition is that the stiffness of the material decreases which has been seen in experimental results $[6,7,11]$. Many authors haye modelled this behaviour with the help of dependency of material parameters on the concentration $[3,12,14,15,18,21]$. There is also a change in the viscoelastic relaxation behaviour with the phase transition, which has been captured by interpolating the relaxation times between the dry and saturated specimen to an effective relaxation time [14,21]. Further, the coupling of the diffusion model on the mechanical loading has been done by making the diffusion coefficient a function of the strain values $[3,15]$. These models have been developed however on a theoretical basis and have not been verified with the

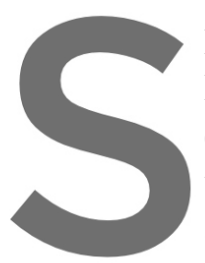
help of experimental resul Experiment conducted ever gave unexpected unloaded specimen. He approach. The model pres
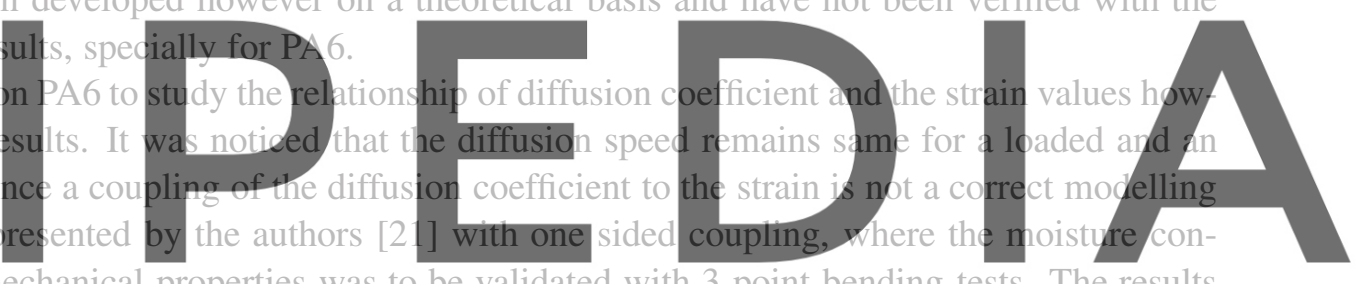

centration affects the mechanical properties was to be validated with 3-point-bending tests. The results

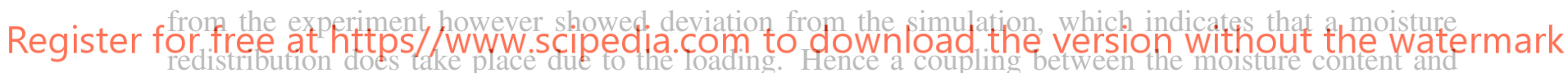

the mechanical loading is indeed important. In the current work, the one sided coupled model has been extended and a strong coupling has been implemented, without effecting the diffusion process directly. A phenomenological approach was taken and instead of moisture redistribution the volume redistribution was considered to calculate the concentrations after loading. In the following sections a short overview of the mechanical and the diffusion model is given, and then the coupling methodology is explained. Finally the results of the fully coupled model is compared with the experimental results.

\section{MODELLING}

A short introduction of the modelling approach for the material behaviour and moisture uptake kinetics is given here. For a more detailed explanation the readers can refer to Sharma et al. [21].

\subsection{Mechanical model}

Polymers exhibit viscoelastic behaviour and PA6 is no exception. It is modelled with the help of a rheological model with four Maxwell elements (Figure 1). The elements are made up of elastic spring 


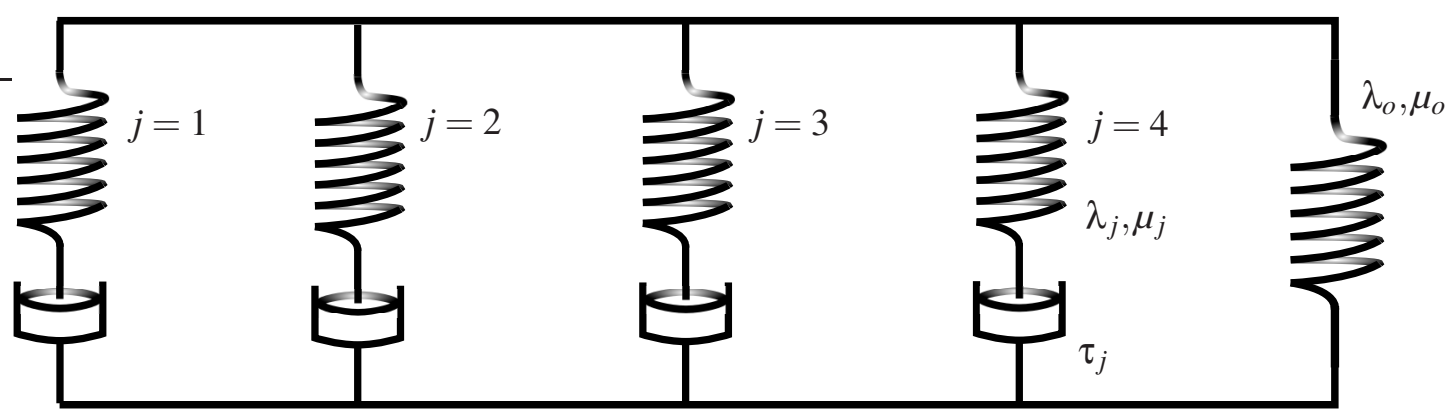

Figure 1: Rheological model for viscoelastic behaviour with four Maxwell arms.

elements with Lamé parameters $\lambda_{j}, \mu_{j}$ for the $j_{t h}$ element. The Maxwell elements are connected to the basic elasticity spring element with Lamé parameters $\lambda_{o}, \mu_{o}$. The relaxation time $\tau_{j}$ for each element is fixed and arranged in power of tens. The shifting of the relaxation spectrum is achieved by modelling the dry material with Maxwell elements of longer relaxation times and the saturated material with the Maxwell elements of shorter relaxation times (Table 1). The rheological model is constrained to small deformations and hence the stress $\sigma$ is calculated with the help of Hooke's law given by,

$$
\sigma=2 \mu_{0} \varepsilon+\lambda_{o} \operatorname{tr} \varepsilon \mathbb{I}+\sum_{j=1}^{4}\left(2 \mu_{j} \varepsilon_{e j}+\lambda_{j} \operatorname{tr} \varepsilon_{e j} \mathbf{I}\right)
$$

\section{Here I is the identity tensc is split additively,
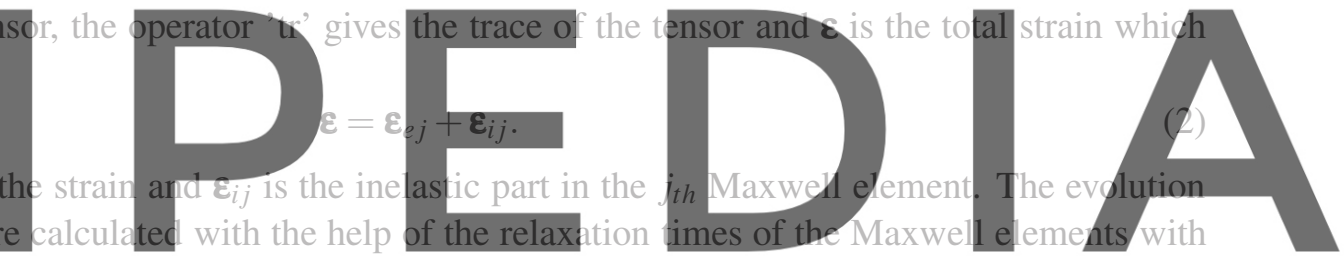

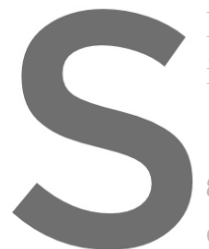

the relation,

Register for free at https//www.scipedia.co|िं to dowwhload the version without the watermark

\subsection{Moisture uptake model}

The moisture uptake in gravimetric experiments showed that the mass of the moisture absorbed increases linearly with the square root of time. This motivates the use of Fick's model which at higher concentration shows a deviation from the experimental results. Hence the diffusion coefficient $D$ is made to vary with the concentration levels, resulting in a non-linear Fick's diffusion model given by,

$$
\frac{d c}{d t}=\operatorname{div}(D(c) \operatorname{grad} c) .
$$

Here $c$ denotes the relative concentration (rel. concentration) of the moisture in the material. The diffusion coefficient is a linear function of $c$, which is given by

$$
D(c)=D_{o}+D_{c} c
$$

where $D_{o}$ and $D_{c}$ are the model parameters. 


\section{COUPLING MECHANICAL STIFFNESSES TO THE CONCENTRATION}

The Lamé parameters for the dry and saturated material were determined with the help of relaxation experiments, where the dry material was modelled with the help of the Maxwell elements with longer relaxation times $(j=2,3,4)$ and the saturated material with the shorter relaxation times $(j=1,2,3)$ (Table 1). The Lamé parameters for any concentration $c$ is calculated with the help of interpolating the

\begin{tabular}{llcccc}
\hline Maxwell element $(\mathrm{j})$ & \multirow{2}{*}{ basic elasticity } & $j=1$ & $j=2$ & $j=3$ & $j=4$ \\
Relaxation time $[\mathrm{s}]$ & & 0.5 & 5 & 50 & 500 \\
\hline Lamé Parameter $\left(\boldsymbol{\lambda}_{d r y}\right)$ Dry $[\mathrm{MPa}]$ & 1901.93 & - & 399.2 & 0.72 & 1.24 \\
\hline Lamé Parameter $\left(\boldsymbol{\lambda}_{\text {wet }}\right)$ Saturated $[\mathrm{MPa}]$ & 270.83 & 359.03 & 446.61 & 172.41 & - \\
\hline
\end{tabular}

Table 1: Lamé parameter $\boldsymbol{\lambda}$ for all Maxwell elements for dry and saturated material. Here $\boldsymbol{\lambda}_{d r y}$ and $\boldsymbol{\lambda}_{\text {wet }}$ denote the vector of all the spring elements for dry and saturated material respectively. Since the Poisson's ratio for the material is unaffected from the moisture content, only $\lambda$ is listed and $\mu$ can be calculated with the Poisson's ratio determined through experiments [21].

two extreme values of dry $\left(\boldsymbol{\lambda}_{d r y}\right)$ and saturated $\left(\boldsymbol{\lambda}_{\text {wet }}\right)$ for all Maxwell elements $(j=1 \ldots 4)$ including the basic elasticity. An interpolation function $f(c)$ needs to be chosen so the interpolation,

$$
\boldsymbol{\lambda}(c)=f(c) \boldsymbol{\lambda}_{d r y}+(1-f(c)) \boldsymbol{\lambda}_{w e t}
$$

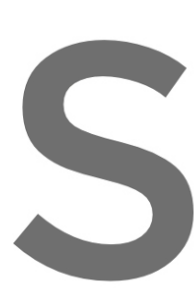

can be done. The interpolation

points, which was not idea

a glassy to rubbery phase.

This measurement was done by

(DSC) and gives the variation

measurement shows that the
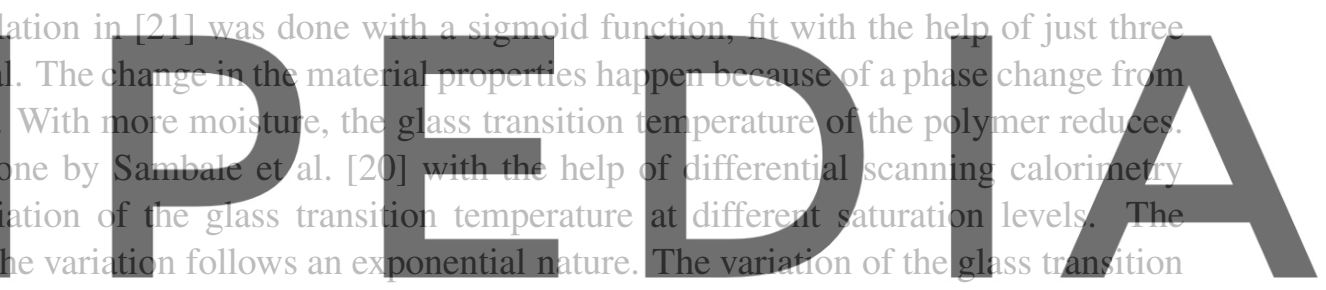

with saturation level was normalised in the range of zero to one, which is used to interpolate the Lamé

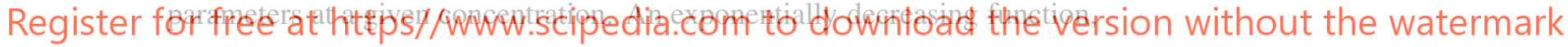

$$
f(c)=\exp (-5 c)
$$

was used to fit the curve between normalised glass transition temperature and the rel. concentration (Figure 2).

\section{COUPLING CONCENTRATION TO THE APPLIED LOADING}

The coupling of the diffusion model is generally done with the help of coupling the diffusion coefficient with the strain value. This is motivated from the fact that under tensile loading the free volume will increase and under compressive loading the free volume will decrease. The changing free volume will affect the speed of moisture uptake and hence the diffusion coefficient should change with the strain values. However in an experiment conducted at LKT, Technical University of Dortmund it was found out that the loading doesn't effect the diffusion speed. 

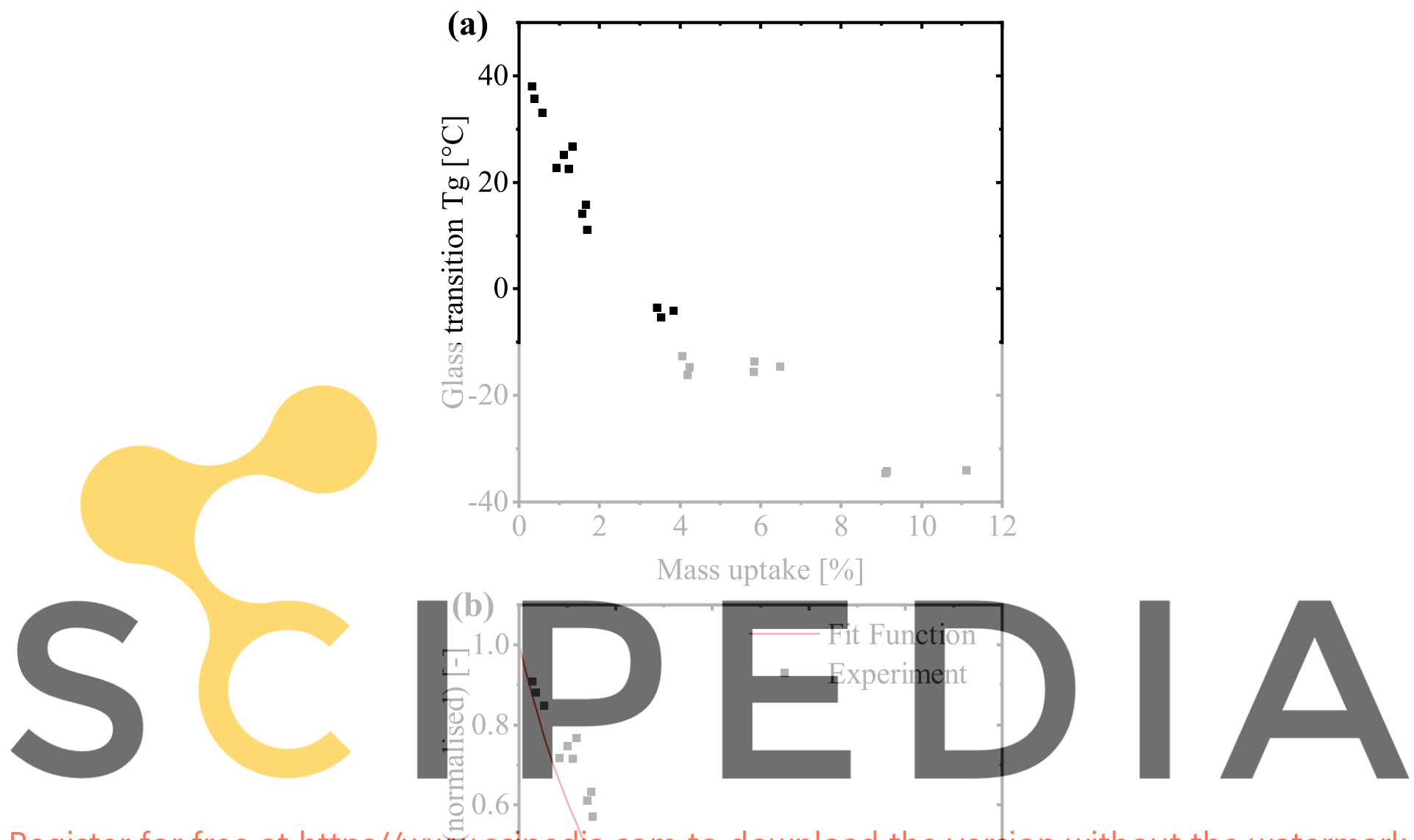

Register for free at https//wWIJ.scipedia.com to download the ver\$ion without the watermark

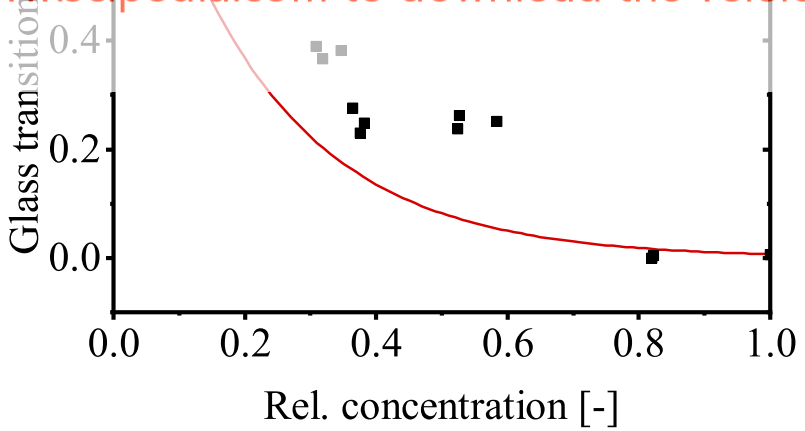

Figure 2: (a) Glass transition temperature for different moisture uptake as weight percentages. (b) The glass transition temperature normalised and fit with decreasing exponential curve. 


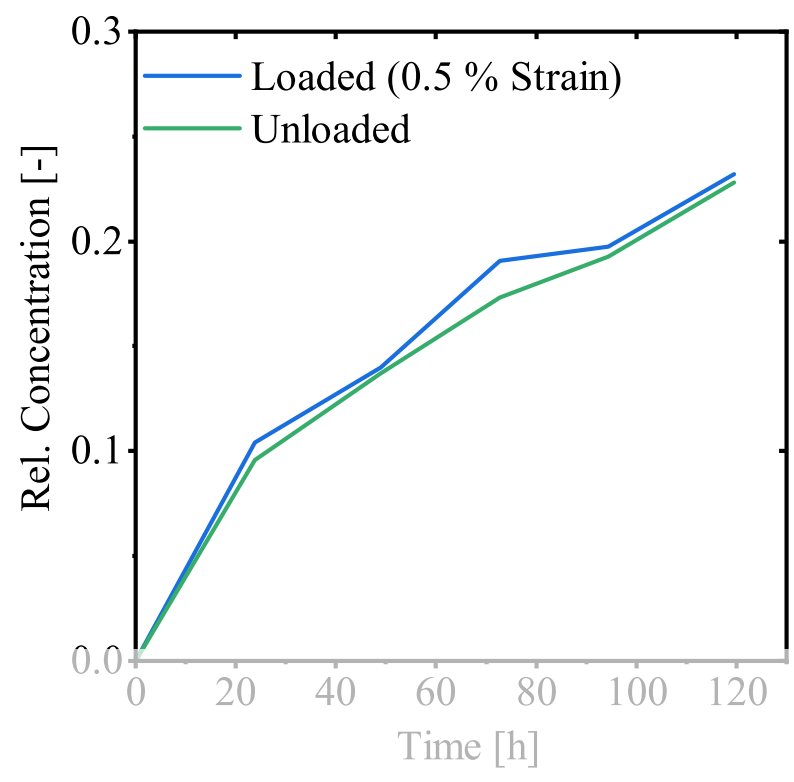

Figure 3: Loaded and unloaded material showing the same diffusion rate.

4.1 Diffusion under loading

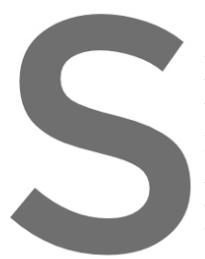

An inhouse relaxation Each of the 12 samples

maintaining the relative

ples were clamped under

the other set was not loaded at a
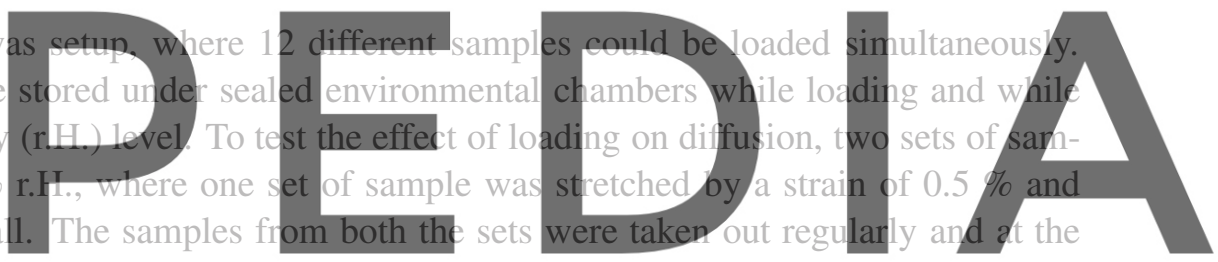

same time (after $25 \mathrm{~h}, 50 \mathrm{~h}, 75 \mathrm{~h}, 100 \mathrm{~h}$ and $125 \mathrm{~h}$ ) to weigh them. The sample taken out from the loaded

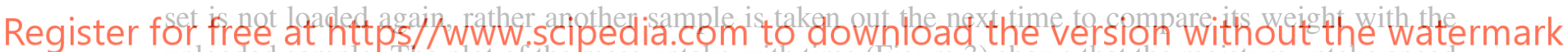
unloaded sample. The plot of the mass uptake with time (Figure 3) shows that the moisture uptake speed

for both sets of the samples were the same. This suggests that the diffusion process is not dependent on the mechanical loading. The assumption that the free volume changes and hence the diffusion speed changes may not hold, as the available integral free volume remains the same. However it is worth mentioning that the experiment was done for small deformation and at higher deformation values the results may differ. Since PA6 is usually used in the regions of geometric linear deformation, experiments at higher deformation were not conducted.

\subsection{3-point-bending experiments}

In order to validate the model under a moisture gradient 3-point bending (3PB) tests were conducted. The tests were conducted on $50 \%$ saturated material. A specimen of the size $40 \mathrm{~mm} \times 4 \mathrm{~mm} \times 2$ $\mathrm{mm}$ was used for the experiments, where the loading was applied on the face with the dimensions 40 $\mathrm{mm} \times 4 \mathrm{~mm}$. In this way the concentration gradient on the specimen along the $2 \mathrm{~mm}$ edge was more dominant than the gradient across the $4 \mathrm{~mm}$ length (Figure 4). The beam was bent until $100 \%$ of the 


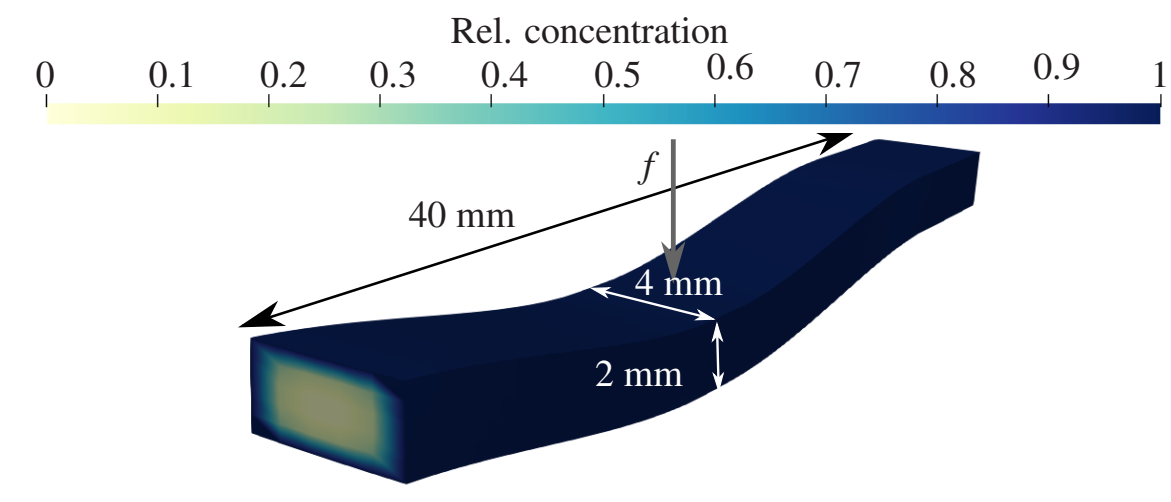

Figure 4: Load applied on the $40 \mathrm{~mm} \times 4 \mathrm{~mm}$ face, so the concentration gradient along the $2 \mathrm{~mm}$ edge is more dominant.
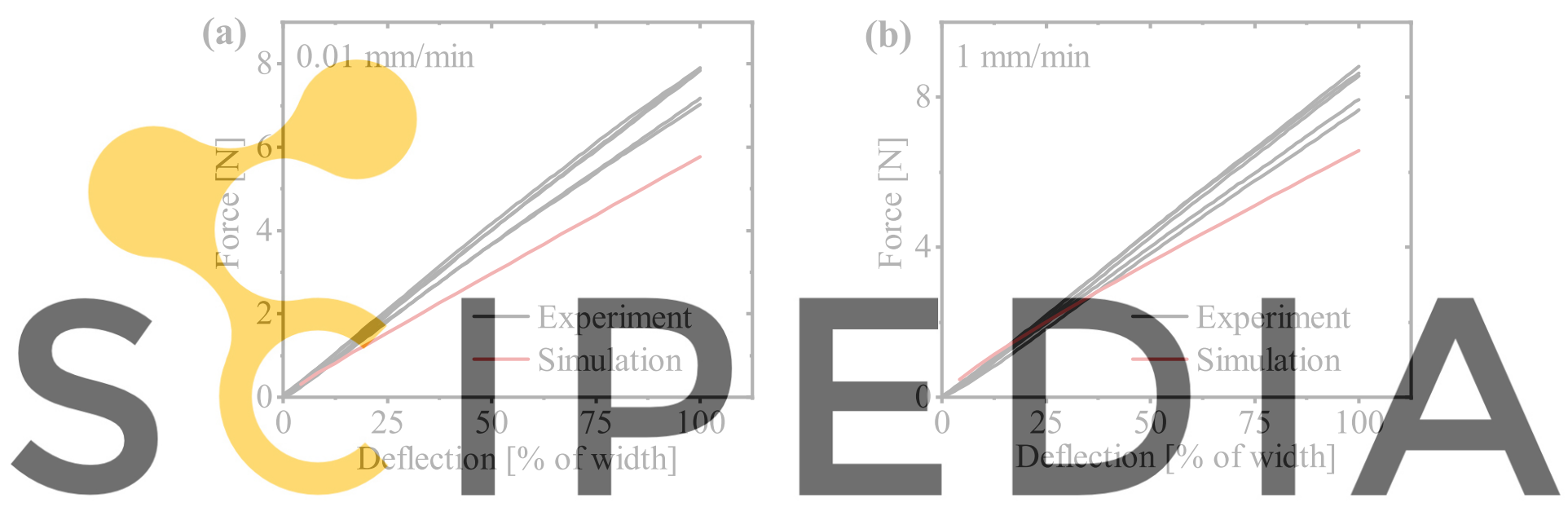

Figure 5: A comparison of the simulation and experimental results with one sided coupled model at $50 \%$ satura-

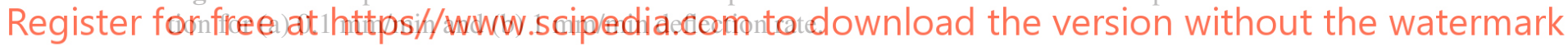

thickness ( $2 \mathrm{~mm}$ ) at two different rates of $0.1 \mathrm{~mm} / \mathrm{min}$ and $1 \mathrm{~mm} / \mathrm{min}$. The tests were conducted in the dynamic mechanical analysis (DMA) Gabo Eplexor 500N machine (Netzsch, Deutschland). The results of the $3 \mathrm{~PB}$ are compared to the simulation results of the one sided coupled model. The vertical force in the bending direction is used to compare the simulation and experimental results, as the stress value is highly dependent on the location of the neutral axis which can wander due to the inhomogeneity caused by moisture distribution.

Although the simulation can reproduce the results until $25 \%$ of the total deflection, there is a notable deviation at higher deflection levels. The simulation results are less stiff than the experimental results as can be seen in Figure 5. This difference in the results are suspected to be a result of moisture redistribution due to the applied loading. Due to the pressure applied by the 3PB stamp on the beam, the moisture must be squeezed out and redistributed in the thickness. It can also be seen that for the slower deflection rate, the simulation shows more deviation than the faster rate. This shows that as the specimen gets more time under loading, the redistribution is more prominent. Thus, a coupling between the load applied and moisture transport does exist. 

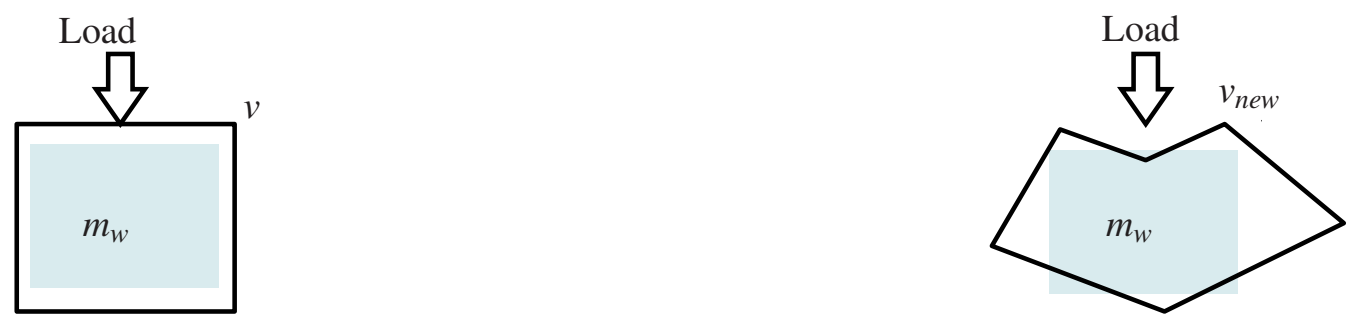

Figure 6: If the mass of water $m_{w}$ in an element of volume $v$ is subjected to loading, then the change in the volume to $v_{\text {new }}$ is used to calculate the new concentration $m_{w} / v_{\text {new }}$.

The duration of the $3 \mathrm{~PB}$ at the slowest deflection rate is just $1200 \mathrm{~s}$. The process of diffusion is too slow to show an effect in this duration and the results in section 4.1 has shown that the diffusion process is independent of the loading. Hence, it can be postulated that the moisture redistribution occurs due to convection.

In order to simulate this behaviour, the change of the volume is used to model the transport. As the concentration is calculated by mass per volume, a transport or change of the volume results in a change of the concentration (Figure 6). This is done instead of introducing diffusion-convection equation for several reasons. Firstly, the numerical stability of a coupled model with a convection-diffusion equation is not reliable. Secondly, the flow of moisture due to convection cannot be restricted to a range of dry $(c=0)$ and fully saturated $(c=1)$ concentrations. Moreover, the velocity driving the convection has

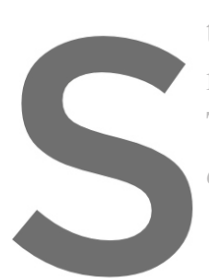
to be calculated with the

model a 3-way coupled pro

The volumetric strain (

$c_{\text {new. }}$. The volumetric strair

and the new rel. concentration is given by,
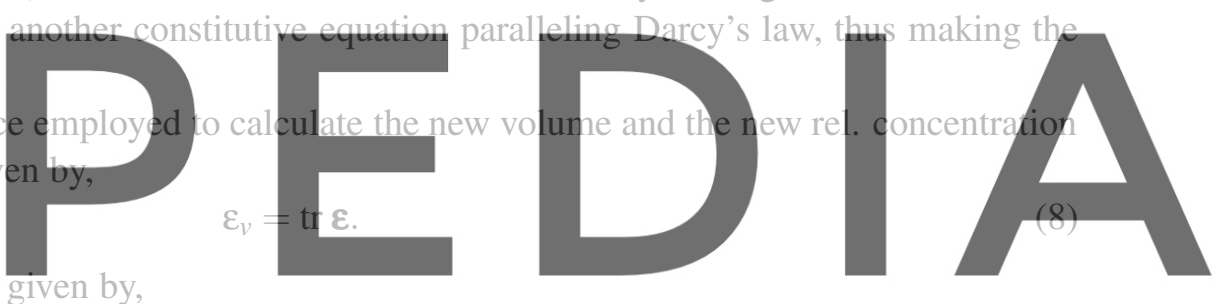

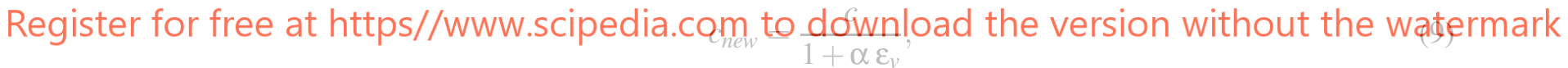

where $\alpha$ is a model parameter. The correction to the rel. concentration is done after solving the set of equations for the one sided model in a seperate step. It doesn't effect the Jacobi matrix for the coupled model, but still introduces a change in the concentration due to loading.

\section{RESULTS WITH COUPLING}

The difference in the moisture distribution with and without coupling can be seen in Figure 7 . With the coupled model, the volume of the upper part of the beam is compressed leading to higher rel. concentration, whereas in the one sided coupled model the distribution is symmetric along the beam thickness (Figure 7).

The force vs. deflection curve also shows a better correlation than the one without coupling (Figure 5). The reason for the better match is that a much larger portion of the width has lesser concentration than the one predicted by the one sided coupled model. Due to the decrease in the moisture content, the force value starts to increase after a certain amount of deflection. It can be seen that the force keeps on increasing with increasing deflection, since the concentration keeps on decreasing until it has no moisture along 

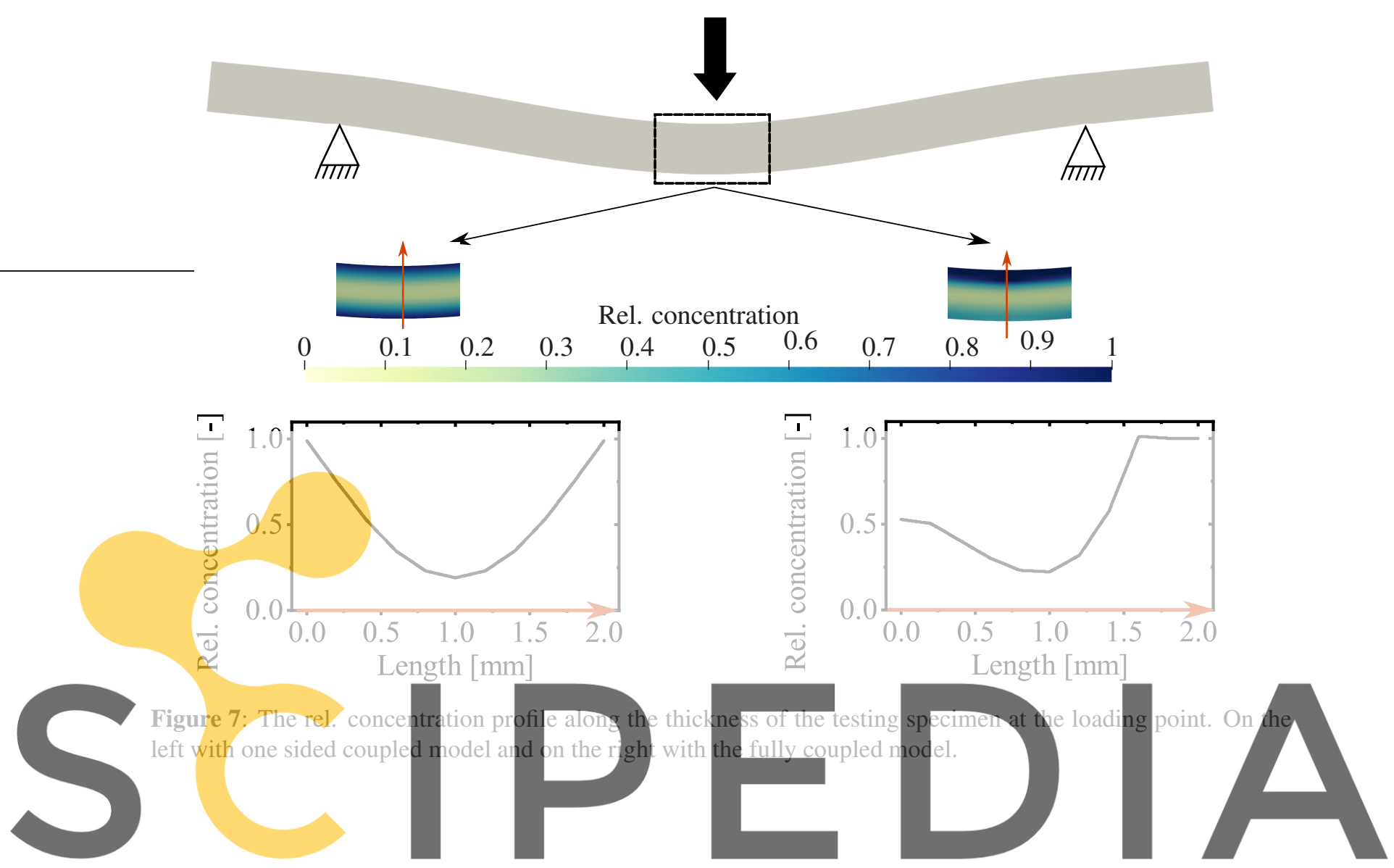

Register for(firee af httṕs/fwww.scipedia.com to dowhlogd the version without the watermark
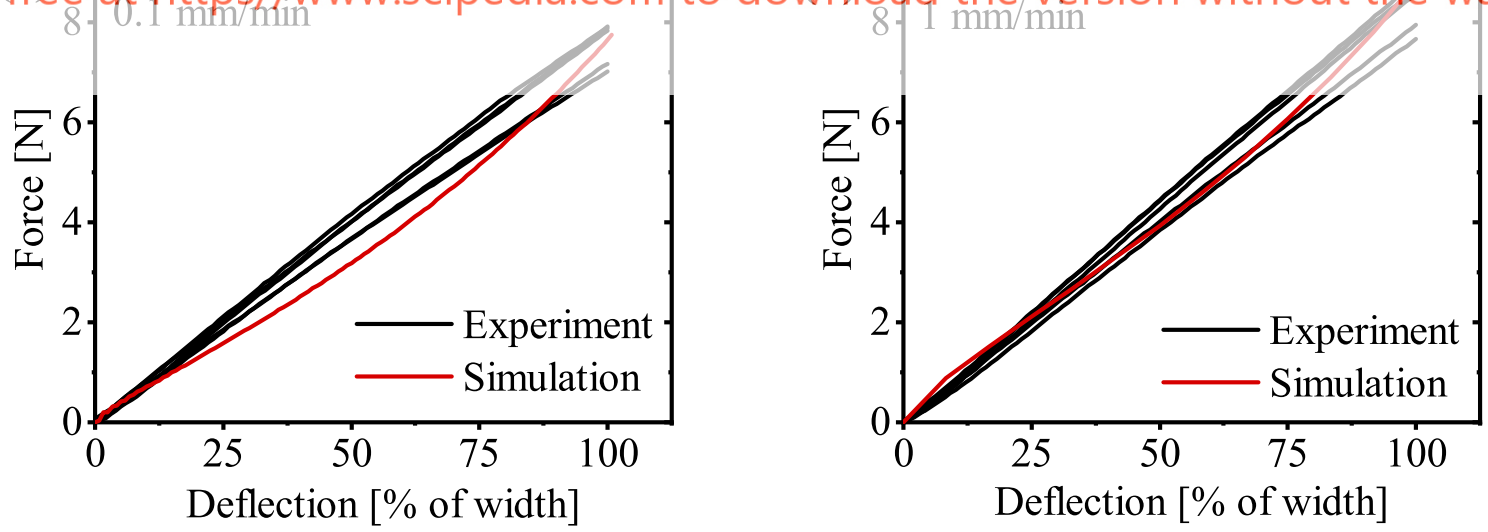

Figure 8: A comparison of the simulation and experimental results with two sided coupled model at $50 \%$ saturation for (a) $0.1 \mathrm{~mm} / \mathrm{min}$ and (b) $1 \mathrm{~mm} / \mathrm{min}$ deflection rate. 
the width. This is also the case with the experimental results. However, in the case of the $1 \mathrm{~mm} / \mathrm{min}$ simulation, this increase is more drastic than experiment, which can be altered with the parameter $\alpha$. For a more accurate result, a convection term should be introduced, which as stated earlier leads to various numerical difficulties.

\section{CONCLUSIONS}

A material model that couples the mechanical deformation with the rel. concentration is presented here. A linear viscoelastic material model is used whose stiffness parameters are dependent on the rel. concentration of the moisture. The relationship between stiffness parameters and the rel. concentration is derived from the glass transition variation. The reverse coupling of the concentration on the loading is done by calculating the corrected volume and adjusting the rel. concentration according to the current volume. The volumetric part of the strain is used to calculate the current volume and hence the concentration.

3-point-bending tests were used to validate the model. A comparison of the one sided coupled model with the experimental result shows that the simulation can match the experiment up to a certain value but with an increase in the deflection the experiment gives higher force values or a stiffer material. This can be attributed to the loss of moisture with the loading. With a two way coupled model the simulation can represent the experimental values more accurately. There is scope for improvement of the material model by introducing a pressure term that comes from the stress applied on the material and which induces a transport of the moisture already absorbed by the material.

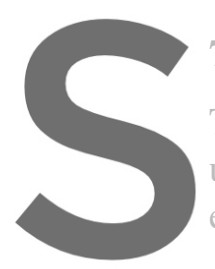

7 ACKNOWLEDGHMENT

The authors are grateful

under Project number Di 4

experiments.
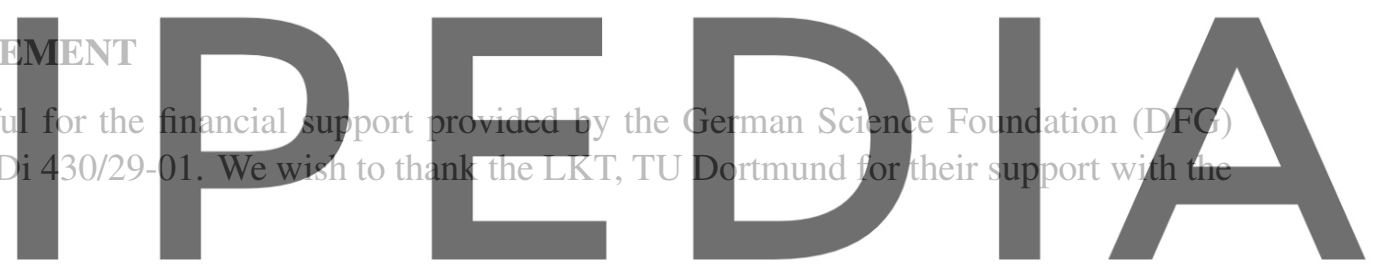

REFERENCES

Register for free at https//Nwww scipedia.com to download the version without the watermark nanocomposites. Express Polymer Letters, 3(4):245-255, 2009.

[2] M. Arhant, P. Le Gac , M. Le Gall, C. Burtin, C. Briançon, and P Davies. Modelling the non Fickian water absorption in polyamide 6. Polymer Degradation and Stability, 133:404-412, nov 2016.

[3] M. Bailakanavar, J. Fish, V. Aitharaju, and W. Rodgers. Computational coupling of moisture diffusion and mechanical deformation in polymer matrix composites. International Journal for Numerical Methods in Engineering, 98(12):859-880, jun 2014.

[4] A. Benaarbia, A. Chrysochoos, and G. Robert. Influence of relative humidity and loading frequency on the PA6.6 cyclic thermomechanical behavior: Part I. mechanical and thermal aspects. Polymer Testing, 40:290-298, dec 2014.

[5] M. Bonnet. Kunststoffe in der Ingenieuranwendung. Springer, 2009.

[6] I. Boukal. Effect of water on the mechanism of deformation of nylon 6. Journal of Applied Polymer Science, 11(8):1483-1494, 1967. 
[7] I. Boukal. Deformationsprozesse und die Struktur von Kristallinen Polymeren. Angewandte Chemie, 74(15):569-573, 1962.

[8] M. Broudin, P. Le Gac, V. Le Saux, C. Champy, G. Robert, P. Charrier, and Y. Marco. Water diffusivity in PA66: Experimental characterization and modeling based on free volume theory. European Polymer Journal, 67:326-334, 2015.

[9] I. Carrascal, J. A. Casado, J. A. Polanco, and F. Gutiérrez-Solana. Absorption and diffusion of humidity in fiberglass-reinforced polyamide. Polymer Composites, 26(5):580-586, 2005.

[10] S. Diebels and A. Geringer. Modelling Inhomogeneous Mechanical Properties in Adhesive Bonds. The Journal of Adhesion, 88(11-12):924-940, dec 2012.

[11] A. Galeski, A. S. Argon, and R. E. Cohen. Changes in the morphology of bulk spherulitic nylon 6 due to plastic deformation. Macromolecules, 21(9):2761-2770, 1988.

[12] F. Goldschmidt and S. Diebels. Modelling and numerical investigations of the mechanical behavior of polyurethane under the influence of moisture. Archive of Applied Mechanics, 85(8):1035-1042, aug 2015.

[13] H. K. Reimschuessel. Relationships on the effect of water on glass transition temperature and young's modulus of nylon 6. Journal of Polymer Science, 16(6):1229-1236, 1978.

[14] M. Johlitz, S. Diebels, and W. Possart. Investigation of the thermoviscoelastic material behaviour of adhesive bonds close to the glass transition temperature. Archive of Applied Mechanics, 82(8):1089-1102, aug 2012.

[15] D. Klepach and T. I. Zohdi. Strain assisted diffusion: Modeling and simulation of deformationdependent diffusion in composite media. Composites Part B: Engineering, 56:413-423, jan 2014.

[16] L. Lim, I. J. Britt, and M. A. Tung. Sorption and transport of water vapor in nylon 6,6 film. Journal of Applied Polymer Science, 71(2):197-206, jan 1999.

[17] K. Nagamatsu. On the viscoelastic properties of crystalline high polymers. Kolloid-Zeitschrift, 172(2):141, 1960.

[18] F. Neff, A. Lion, and M. Johlitz. Modelling diffusion induced swelling behaviour of natural rubber in an organic liquid, ZAMM-Journal of Applied Mathematics and Mechanics/Zeitschrift für Angewandte Mathematik und Mechanik, 99(3), mar 2019.

[19] R. Puffr and J. Šebenda. On the structure and properties of polyamides. XXVII. The mechanism of water sorption in polyamides. In Journal of Polymer Science: Polymer Symposia, volume 16, pages 79-93. Wiley Online Library, 1967.

[20] A. Sambale, M. Kurkowski, and M. Stommel. Determination of moisture gradients in polyamide 6 using StepScan DSC. Thermochimica Acta, 672:150-156, feb 2019.

[21] P. Sharma, A. Sambale, M. Stommel, M. Maisl, H. G. Herrmann, and S. Diebels. Moisture transport in PA6 and its influence on the mechanical properties. Continuum Mechanics and Thermodynamics, 32(2):307-325, mar 2020.

[22] R. Siddique, J. Khatib, and I. Kaur. Use of recycled plastic in concrete: A review. Waste Management, 28(10):1835-1852, 2008. 
[23] N. Strumberger, D. Se, and F. Zagreb. Polymeric Materials in Automobiles. Promet-Traffic \& Transportation, 17(3):149-160, 2005.

[24] R. A. Witik, J. Payet, V. Michaud, C. Ludwig, and J. E. Månson. Assessing the life cycle costs and environmental performance of lightweight materials in automobile applications. Composites Part A: Applied Science and Manufacturing, 42(11):1694-1709, nov 2011.

[25] J. Wortberg, F. Heinzler, J. Dörner, and K. Saul. Innovative Kunststoffanwendungen im Automobil. In Herausforderungen für das Automotive Engineering \& Management, pages 107-122. Springer Fachmedien Wiesbaden, 2013. 